\title{
BVPs for higher-order integro-differential equations with $\phi$-Laplacian and functional boundary conditions
}

\author{
Xiufeng Guo ${ }^{1}$, Liang Lu ${ }^{1,2^{*}}$ and Zexian Liu ${ }^{1}$
}

"Correspondence:

gxluliang@163.com

${ }^{1}$ College of Sciences, Hezhou

University, Hezhou, Guangxi

542899, People's Republic of China

${ }^{2}$ Guangxi Key Laboratory of Hybrid

Computation and IC Design

Analysis, Nanning, Guangxi 530006,

People's Republic of China

\begin{abstract}
In this paper, we study the existence of solutions of a class of higher-order integro-differential boundary value problems with $\phi$-Laplacian like operator and functional boundary conditions. By giving the definition of a pair of coupled lower and upper solutions and some new hypotheses, we obtain some new existence results for boundary value problems with $\phi$-Laplacian like operator by employing the Schauder fixed point theorem and an appropriate Nagumo condition. Finally, an example is given to illustrate the results.
\end{abstract}

MSC: 39K10; 34B15

Keywords: $\phi$-Laplacian like operator; integro-differential equation; functional boundary condition; Nagumo condition; lower and upper solutions

\section{Introduction}

Integro-differential equations have become more and more important in some mathematical models of real phenomena, especially in control, biological, medical, and informational models. Boundary value problems (BVPs) for nonlinear integro-differential equations are used to describe a great number of nonlinear phenomena in science (see $[1,2])$, moreover, the theory of $\phi$-Laplacian BVPs has emerged as an important area in recent years (see [3-6]). In this paper, we will consider the following BVPs of higher-order functional integro-differential $\phi$-Laplacian like equations with functional boundary conditions:

$$
\begin{aligned}
& {\left[\phi\left(u^{(n-1)}(t)\right)\right]^{\prime}+A u(t)=0, \quad t \in J^{\prime},} \\
& \left\{\begin{array}{l}
g_{i}\left(u, W_{0} u, S_{0} u, \ldots, u^{(n-1)}, W_{n-1} u^{(n-1)}, S_{n-1} u^{(n-1)}, u^{(i)}(0)\right)=0, \quad i=0, \ldots, n-2, \\
g_{n-1}\left(u, W_{0} u, S_{0} u, \ldots, u^{(n-1)}, W_{n-1} u^{(n-1)}, S_{n-1} u^{(n-1)}, u^{(n-2)}(T)\right)=0,
\end{array}\right.
\end{aligned}
$$

where $n \geq 2$ is an integer, $\phi$ is an increasing homeomorphism operator,

$$
\begin{aligned}
A u(t)= & f\left(t, u(t), u\left(\alpha_{0}(t)\right), W_{0} u(t), S_{0} u(t), u^{\prime}(t), u^{\prime}\left(\alpha_{1}(t)\right), W_{1} u^{\prime}(t), S_{1} u^{\prime}(t), \ldots,\right. \\
& \left.u^{(n-1)}(t), u^{(n-1)}\left(\alpha_{n-1}(t)\right), W_{n-1} u^{(n-1)}(t), S_{n-1} u^{(n-1)}(t)\right),
\end{aligned}
$$

O2014 Guo et al.; licensee Springer. This is an Open Access article distributed under the terms of the Creative Commons Attribution License (http://creativecommons.org/licenses/by/2.0), which permits unrestricted use, distribution, and reproduction in any medium, provided the original work is properly cited. 
and $J=[0, T](T>0), J^{\prime}=(0, T)$, for $i=0,1, \ldots, n-1, g_{i}:(C[0, T])^{3 n} \times R \rightarrow R$ are continuous functions, and for $l=0,1, \ldots, n-1$,

$$
W_{l} u^{(l)}(t)=\int_{0}^{\beta_{l}(t)} k_{l}(t, s) u^{(l)}\left(\gamma_{l}(s)\right) d s, \quad S_{l} u^{(l)}(t)=\int_{0}^{T} h_{l}(t, s) u^{(l)}\left(\delta_{l}(s)\right) d s
$$

$k_{l}(t, s) \in C\left(D_{l}, R^{+}\right), D_{l}=\left\{(t, s) \in R^{2}: t \in J, 0 \leq s \leq \beta_{l}(t)\right\}, h_{l}(t, s) \in C\left(J \times J, R^{+}\right), R^{+}=[0,+\infty)$, $\alpha_{l}, \beta_{l}, \gamma_{l}, \delta_{l} \in C(J, J)$, and $f: J^{\prime} \times R^{4 n} \rightarrow R$ is a Carathéodory function, that is:

(i) for any $\left(x_{0}, \ldots, x_{4 n-1}\right) \in R^{4 n}, f\left(t, x_{0}, \ldots, x_{4 n-1}\right)$ is measurable on $J^{\prime}$,

(ii) for a.e. $t \in J^{\prime}, f(t, \cdot, \ldots, \cdot)$ is continuous on $R^{4 n}$,

(iii) for every compact set $K \subset R^{4 n}$, there exists a nonnegative function $\mu_{K}(t) \in L^{1}(0, T)$ such that

$$
\left|f\left(t, x_{0}, \ldots, x_{4 n-1}\right)\right| \leq \mu_{K}(t), \quad\left(t, x_{0}, \ldots, x_{4 n-1}\right) \in J^{\prime} \times K
$$

We say $u(t)$ is a solution of BVP (1.1) and (1.2), that is, a function $u(t) \in C^{n-1}[0, T]$ such that $\phi\left(u^{(n-1)}\right)$ is absolutely continuous on $J^{\prime}, u(t)$ satisfies (1.1) a.e. on $J^{\prime}$, and $u(t)$ satisfies boundary condition (1.2).

As we know, higher-order boundary value problems for differential equations have received great attention in recent years (see [7-16]). We found that BVP (1.1) and (1.2) is more general in the literature, and the functional boundary condition (1.2) may not only cover many classical boundary conditions, such as various linear two-point, multi-point studied by many authors, but it may also include many new boundary conditions not studied so far in the literature. In recent years, BVPs with linear and nonlinear boundary conditions have been extensively investigated by numerous researchers. For a small sample of such work, we refer the reader to [17-26]. As is well known, a variety of methods and tools, such as lower and upper solution methods and various fixed point theorems, are very useful and have been successfully used to prove the existence of solutions of BVPs.

Motivated by the above mentioned works, we consider the BVPs of higher-order functional integro-differential equations (1.1) and (1.2) with $\phi$-Laplacian like operator and functional boundary conditions in this paper. As we know, BVP (1.1) and (1.2) has not yet been considered. By introducing a definition for the coupled lower and upper solutions of BVP (1.1) and (1.2), we obtain the existence of solutions of the problem based on the assumption that there exists a pair of coupled lower and upper solutions.

This paper is organized as follows. In Section 2, we state some preliminaries and lemmas which will be used throughout this paper. In Section 3, some results concerning coupled lower and upper solutions are given. Finally, an example is given to illustrate our results in Section 4.

\section{Preliminaries}

Throughout this paper, let $E=C^{n-1}[0, T],\|u\|_{\infty}=\max \{|u(t)|: t \in J\}$ for any $u \in C[0, T]$, and

$$
\|u\|=\max \left\{\|u\|_{\infty},\left\|u^{\prime}\right\|_{\infty}, \ldots,\left\|u^{(n-1)}\right\|_{\infty}\right\}
$$


and

$$
\|u\|_{p}= \begin{cases}\left(\int_{0}^{T}|u(t)|^{p} d t\right)^{\frac{1}{p}}, & 1 \leq p<\infty \\ \inf \{M: \mu(\{t:|u(t)|>M\})=0\}, & p=\infty\end{cases}
$$

stand for the norms in $E$ and $L^{p}(0, T)$, respectively, where $\mu(\cdot)$ denotes the Lebesgue measure of a set. In what follows, a functional $y: C[0, T] \rightarrow R$ is said to be nondecreasing if $y\left(u_{1}\right) \geq y\left(u_{2}\right)$ for any $u_{1}, u_{2} \in C[0, T]$ with $u_{1}(t) \geq u_{2}(t)$ on $[0, T]$. A similar definition holds for $y$ to be non-increasing.

Definition 2.1 Let $f: J^{\prime} \times R^{4 n} \rightarrow R$ be Carathéodory function, and $v, w \in E$ satisfy

$$
v^{(i)}(t) \leq w^{(i)}(t), \quad t \in J, i=0,1, \ldots, n-2 .
$$

We say that $f$ satisfies the Nagumo condition with respect to $v$ and $w$ if for

$$
\xi=\max \left\{\frac{w^{(n-2)}(T)-v^{(n-2)}(0)}{T}, \frac{w^{(n-2)}(0)-v^{(n-2)}(T)}{T}\right\},
$$

there exists a constant $C=C(v, w)$ with

$$
C>\max \left\{\xi,\left\|v^{(n-1)}\right\|_{\infty},\left\|w^{(n-1)}\right\|_{\infty}\right\}
$$

and functions $\psi \in C[0, \infty), \vartheta \in L^{p}(0, T)(1 \leq p \leq \infty)$, such that $\psi>0$ on $[0, \infty)$,

$$
\left|f\left(t, x_{0}, \ldots, x_{4 n-1}\right)\right| \leq \vartheta(t) \psi\left(\left|x_{4(n-1)}\right|\right) \quad \text { on } J^{\prime} \times \mathbb{D}_{v}^{w} \times R^{4}
$$

and

$$
\int_{\phi(\xi)}^{\phi(C)} \frac{\left(\phi^{-1}(x)\right)^{(p-1) / p}}{\psi\left(\left|\phi^{-1}(x)\right|\right)} d x, \quad \int_{\phi(-C)}^{\phi(-\xi)} \frac{\left(\phi^{-1}(x)\right)^{(p-1) / p}}{\psi\left(\left|\phi^{-1}(x)\right|\right)} d x>\|\vartheta\|_{p} \zeta^{(p-1) / p}
$$

where $(p-1) / p \equiv 1$ for $p=\infty$,

$$
\begin{aligned}
\mathbb{D}_{v}^{w}= & {[v(t), w(t)] \times\left[v\left(\alpha_{0}(t)\right), w\left(\alpha_{0}(t)\right)\right] \times\left[W_{0} v(t), W_{0} w(t)\right] \times\left[S_{0} v(t), S_{0} w(t)\right] \times \cdots } \\
& \times\left[v^{(n-2)}(t), w^{(n-2)}(t)\right] \times\left[v^{(n-2)}\left(\alpha_{n-2}(t)\right), w^{(n-2)}\left(\alpha_{n-2}(t)\right)\right] \\
& \times\left[W_{n-2} v^{(n-2)}(t), W_{n-2} w^{(n-2)}(t)\right] \times\left[S_{n-2} v^{(n-2)}(t), S_{n-2} w^{(n-2)}(t)\right]
\end{aligned}
$$

and

$$
\zeta=\max _{t \in J} w^{(n-2)}(t)-\min _{t \in J} v^{(n-2)}(t)
$$

Remark 2.1 Let $v, w \in E$ satisfy (2.1). Assume that there exist $\theta \in L^{p}(0, T), 1 \leq p \leq \infty$, and $\sigma \in[0, \infty)$ such that

$$
\left|f\left(t, x_{0}, \ldots, x_{4 n-1}\right)\right| \leq \theta(t)\left(1+\left|x_{4(n-1)}\right|^{\sigma}\right) \quad \text { on } J^{\prime} \times \mathbb{D}_{v}^{w} \times R^{4}
$$

Then $f$ satisfies the Nagumo condition with respect to $v$ and $w$ with $\psi(x)=1+|x|^{\sigma}$. 
Definition 2.2 Let $C$ be the constant introduced in Definition 2.1. Assume that there exist $v, w \in E$ satisfying (2.1), $\phi\left(v^{(n-1)}\right)$ and $\phi\left(w^{(n-1)}\right)$ are absolutely continuous on $J^{\prime}$. Then $v$ and $w$ are said to be a pair of coupled lower and upper solutions of BVP (1.1) and (1.2) if

$$
\begin{gathered}
{\left[\phi\left(v^{(n-1)}(t)\right)\right]^{\prime}+f\left(t, v(t), v\left(\alpha_{0}(t)\right), W_{0} v(t), S_{0} v(t), \ldots, v^{(n-2)}(t), v^{(n-2)}\left(\alpha_{n-2}(t)\right),\right.} \\
\left.W_{n-2} v^{(n-1)}(t), S_{n-2} v^{(n-2)}(t),-C,-C,-W_{n-1} C,-S_{n-1} C\right) \geq 0 \quad \text { a.e. } t \in J^{\prime}, \\
\left\{\begin{array}{c}
\min _{\|z\|_{\infty} \leq C} g_{i}\left(v, W_{0} v, S_{0} v, \ldots, v^{(n-2)}, W_{n-2} v^{(n-1)}, S_{n-2} v^{(n-2)},\right. \\
\left.z, W_{n-1} z, S_{n-1} z, v^{(i)}(0)\right) \geq 0, \quad i=0, \ldots, n-2, \\
\min _{\|z\|_{\infty} \leq C} g_{n-1}\left(v, W_{0} v, S_{0} v, \ldots, v^{(n-2)}, W_{n-2} v^{(n-2)}, S_{n-2} v^{(n-2)},\right. \\
\left.z, W_{n-1} z, S_{n-1} z, v^{(n-2)}(T)\right) \geq 0,
\end{array}\right.
\end{gathered}
$$

and

$$
\begin{aligned}
& {\left[\phi\left(w^{(n-1)}(t)\right)\right]^{\prime}+f\left(t, w(t), w\left(\alpha_{0}(t)\right), W_{0} w(t), S_{0} w(t), \ldots, w^{(n-2)}(t), w^{(n-2)}\left(\alpha_{n-2}(t)\right),\right.} \\
& \left.W_{n-2} w^{(n-1)}(t), S_{n-2} w^{(n-2)}(t), C, C, W_{n-1} C, S_{n-1} C\right) \leq 0 \quad \text { a.e. } t \in J^{\prime}, \\
& \left\{\begin{array}{c}
\max _{\|z\|_{\infty} \leq C} g_{i}\left(w, W_{0} w, S_{0} w, \ldots, w^{(n-2)}, W_{n-2} w^{(n-2)}, S_{n-2} w^{(n-2)},\right. \\
\left.z, W_{n-1} z, S_{n-1} z, w^{(i)}(0)\right) \leq 0, \quad i=0, \ldots, n-2, \\
\max _{\|z\|_{\infty} \leq C} g_{n-1}\left(w, W_{0} w, S_{0} w, \ldots, w^{(n-2)}, W_{n-2} w^{(n-2)}, S_{n-2} w^{(n-2)},\right. \\
\left.z, W_{n-1} z, S_{n-1} z, w^{(n-2)}(T)\right) \leq 0 .
\end{array}\right.
\end{aligned}
$$

For convenience, we first list the following hypotheses:

(H1) $\phi(x)$ is increasing on $R$;

(H2) BVP (1.1) and (1.2) has a pair of coupled lower and upper solutions $v$ and $w$ satisfying (2.1);

(H3) the functional $f$ satisfies the Nagumo condition with respect to $v$ and $w$;

(H4) for $\left(t, x_{0}, x_{1}, \ldots, x_{4 n-1}\right) \in J^{\prime} \times R^{4 n}$ with $v^{(i)}(t) \leq x_{4 i} \leq w^{(i)}(t)$, $v^{(i)}\left(\alpha_{i}(t)\right) \leq x_{4 i+1} \leq w^{(i)}(t), W_{i} v^{(i)}(t) \leq x_{4 i+2} \leq W_{i} w^{(i)}(t), S_{i} v^{(i)}(t) \leq x_{4 i+3} \leq S_{i} w^{(i)}(t)$, $i=0,1, \ldots, n-3$, and $v^{(n-2)}\left(\alpha_{n-2}(t)\right) \leq x_{4 n-7} \leq w^{(n-2)}(t)$, $W_{n-2} v^{(n-2)}(t) \leq x_{4 n-6} \leq W_{n-2} w^{(n-2)}(t), S_{n-2} v^{(n-2)}(t) \leq x_{4 n-5} \leq S_{n-2} w^{(n-2)}(t)$, we have

$$
\begin{aligned}
& f\left(\cdot, v(t), v\left(\alpha_{0}(t)\right), W_{0} v(t), S_{0} v(t), \ldots, x_{4 n-8}, v^{(n-2)}\left(\alpha_{n-2}(t)\right),\right. \\
&\left.\quad W_{n-2} v^{(n-2)}(t), S_{n-2} v^{(n-2)}(t), x_{4 n-4}, \ldots, x_{4 n-1}\right) \\
& \leq f\left(\cdot, x_{0}, x_{1}, \ldots, x_{4 n-8}, \ldots, x_{4 n-4}, \ldots, x_{4 n-1}\right) \\
& \leq f\left(\cdot, w(t), w\left(\alpha_{0}(t)\right), W_{0} w(t), S_{0} v(t), \ldots, x_{4 n-8}, w^{(n-2)}\left(\alpha_{n-2}(t)\right),\right. \\
&\left.W_{n-2} w^{(n-2)}(t), S_{n-2} w^{(n-2)}(t), x_{4 n-4}, \ldots, x_{4 n-1}\right),
\end{aligned}
$$

and $f\left(t, x_{0}, x_{1}, \ldots, x_{4 n-1}\right)$ is nondecreasing in the arguments $x_{4 n-4}, x_{4 n-3}, x_{4 n-2}$, $x_{4 n-1}$;

(H5) for $i=0,1, \ldots, n-1$ and $\left(y_{0}, y_{1}, \ldots, y_{3 n-1}, z\right) \in(C[0, T])^{3 n} \times R, g_{i}\left(y_{0}, y_{1}, \ldots, y_{3 n-1}, z\right)$ are nondecreasing in the arguments $y_{0}, \ldots, y_{3 n-4}$. 
We assume that conditions (H1)-(H5) hold throughout this paper. For $u \in C^{n-2}[0, T]$ and $i=0,1, \ldots, n-2$, we define

$$
\bar{u}^{[i]}(t)=\max \left\{v^{(i)}(t), \min \left\{u^{(i)}(t), w^{(i)}(t)\right\}\right\},
$$

then, for $i=0,1, \ldots, n-2, \bar{u}^{(i)}(t)$ is continuous on $J$, and

$$
v^{(i)}(t) \leq \bar{u}^{[i]}(t) \leq w^{(i)}(t), \quad \bar{v}^{[i]}(t)=v^{(i)}(t), \quad \bar{w}^{[i]}(t)=w^{(i)}(t),
$$

for $t \in J$ and $i=0,1, \ldots, n-2$. Let $C=C(v, w)$ be the constant introduced in Definition 2.1, and define

$$
\begin{aligned}
& \varphi(x)= \begin{cases}\phi(x), & |x| \leq C, \\
\frac{1}{2 C}(\phi(C)-\phi(-C)) x+\frac{1}{2}(\phi(C)+\phi(-C)), & |x|>C,\end{cases} \\
& \hat{u}^{[n-1]}(t)=\max \left\{-C, \min \left\{u^{(n-1)}(t), C\right\}\right\}, \quad u \in E,
\end{aligned}
$$

and a functional $F: J^{\prime} \times E \rightarrow R$ by

$$
F(t, u(\cdot))=B u(t)+\frac{\bar{u}^{[n-2]}(t)-u^{(n-2)}(t)}{1+\left(u^{(n-2)}(t)\right)^{2}},
$$

where

$$
\begin{aligned}
B u(t)= & f\left(t, \bar{u}^{[0]}(t), \bar{u}^{[0]}\left(\alpha_{0}(t)\right), S_{0} \bar{u}^{[0]}(t), \ldots, \bar{u}^{[n-2]}(t), \bar{u}^{[n-2]}\left(\alpha_{n-2}(t)\right),\right. \\
& W_{0} \bar{u}^{[0]}(t), W_{n-2} \bar{u}^{[n-2]}(t), S_{n-2} \bar{u}^{[n-2]}(t), \hat{u}^{[n-1]}(t), \\
& \left.\hat{u}^{[n-1]}\left(\alpha_{n-1}(t)\right), W_{n-1} \hat{u}^{[n-1]}(t), S_{n-1} \hat{u}^{[n-1]}(t)\right) .
\end{aligned}
$$

Then, in view of (H1) and (2.13), we find that $\varphi: R \rightarrow R$ is increasing and continuous (hence $\varphi^{-1}$ exists), and

$$
\lim _{x \rightarrow-\infty} \varphi(x)=-\infty, \quad \lim _{x \rightarrow \infty} \varphi(x)=\infty
$$

What is more, for $u \in E$ and $t \in J^{\prime}, F(t, u(\cdot))$ is continuous in $u$, and we can see that

$$
|F(t, u(\cdot))| \leq \vartheta(t) \max _{z \in[0, C]} \psi(z)+\|v\|+\|w\|+1 .
$$

Now, we consider the BVP consisting of the equation

$$
\left[\varphi\left(u^{(n-1)}(t)\right)\right]^{\prime}+F(t, u(\cdot))=0, \quad t \in J^{\prime}
$$

and the boundary condition

$$
\left\{\begin{aligned}
u^{(i)}(0)= & g_{i}\left(\bar{u}^{[0]}, W_{0} \bar{u}^{[0]}, S_{0} \bar{u}^{[0]}, \ldots, \bar{u}^{[n-2]}, W_{n-2} \bar{u}^{[n-2]}, S_{n-2} \bar{u}^{[n-2]},\right. \\
& \left.\hat{u}^{[n-1]}, W_{n-1} \hat{u}^{[n-1]}, S_{n-1} \hat{u}^{[n-1]}, \bar{u}^{[i]}(0)\right)+\bar{u}^{[i]}(0), \quad i=0, \ldots, n-2, \\
u^{(n-2)}(T)= & g_{n-1}\left(\bar{u}^{[0]}, W_{0} \bar{u}^{[0]}, S_{0} \bar{u}^{[0]}, \ldots, \bar{u}^{[n-2]}, W_{n-2} \bar{u}^{[n-2]}, S_{n-2} \bar{u}^{[n-2]},\right. \\
& \left.\hat{u}^{[n-1]}, W_{n-1} \hat{u}^{[n-1]}, S_{n-1} \hat{u}^{[n-1]}, \bar{u}^{[n-2]}(T)\right)+\bar{u}^{[n-2]}(T) .
\end{aligned}\right.
$$


Lemma 2.1 For any fixed $u \in E$, define $\mathcal{L}(\cdot ; u): R \rightarrow R$ by

$$
\mathcal{L}(x ; u)=\int_{0}^{T} \varphi^{-1}\left(x-\int_{0}^{\tau} F(s, u(\cdot)) d s\right) d \tau+g_{u}
$$

where

$$
\begin{aligned}
g_{u}= & \bar{u}^{[n-2]}(0)+g_{n-2}\left(\bar{u}^{[0]}, W_{0} \bar{u}^{[0]}, S_{0} \bar{u}^{[0]}, \ldots, \bar{u}^{[n-2]}, W_{n-2} \bar{u}^{[n-2]},\right. \\
& \left.S_{n-2} \bar{u}^{[n-2]}, \hat{u}^{[n-1]}, W_{n-1} \hat{u}^{[n-1]}, S_{n-1} \hat{u}^{[n-1]}, \bar{u}^{[n-2]}(0)\right) \\
& -\bar{u}^{[n-2]}(T)-g_{n-1}\left(\bar{u}^{[0]}, W_{0} \bar{u}^{[0]}, S_{0} \bar{u}^{[0]}, \ldots, \bar{u}^{[n-2]}, W_{n-2} \bar{u}^{[n-2]},\right. \\
& \left.S_{n-2} \bar{u}^{[n-2]}, \hat{u}^{[n-1]}, W_{n-1} \hat{u}^{[n-1]}, S_{n-1} \hat{u}^{[n-1]}, \bar{u}^{[n-2]}(T)\right) .
\end{aligned}
$$

Then the equation

$$
\mathcal{L}(\cdot ; u)=0
$$

has a unique solution.

Proof We first note that $\mathcal{L}(\cdot ; u)$ is continuous and increasing on $R$. From $(2.16)$, we have

$$
\lim _{x \rightarrow-\infty} \mathcal{L}(x ; u)=-\infty \text { and } \quad \lim _{x \rightarrow \infty} \mathcal{L}(x ; u)=\infty
$$

Then, from the fact that $\mathcal{L}(\cdot ; u)$ is continuous and increasing on $R$, a standard argument shows that there exists a unique solution of (2.22).

Lemma 2.2 For $u \in E$, let

$$
P_{n} u(t)=\varphi^{-1}\left(x_{u}-\int_{0}^{t} F(s, u(\cdot)) d s\right)
$$

with $x_{u}$ being the unique solution of $(2.22)$ and $F(s, u(\cdot))$ be defined by (2.15). Then $u(t)$ is a solution of $B V P(2.18)$ and (2.19) if and only if $u(t)$ is a solution of the following equation:

$$
\begin{aligned}
u(t)= & \frac{1}{(n-2) !} \int_{0}^{t}(t-s)^{n-2} P_{n} u(s) d s \\
& +\sum_{i=0}^{n-2} \frac{t^{i}}{i !}\left(\bar{u}^{[i]}(0)+g_{i}\left(\bar{u}^{[0]}, W_{0} \bar{u}^{[0]}, S_{0} \bar{u}^{[0]}, \ldots, \bar{u}^{[n-2]}, W_{n-2} \bar{u}^{[n-2]},\right.\right. \\
& \left.\left.S_{n-2} \bar{u}^{[n-2]}, \hat{u}^{[n-1]}, W_{n-1} \hat{u}^{[n-1]}, S_{n-1} \hat{u}^{[n-1]}, \bar{u}^{[i]}(0)\right)\right), \quad n \geq 2,
\end{aligned}
$$

where we take $0^{0}=1$.

Proof This can be verified by direct computations, so we omit it.

\section{Main results}

In this section, we will state and prove our existence results for BVP (1.1) and (1.2). 
Theorem 3.1 Assume the hypotheses (H1)-(H5) hold. Then BVP (1.1) and (1.2) has at least one solution $u(t)$ satisfying

$$
v^{(i)}(t) \leq u^{(i)}(t) \leq w^{(i)}(t) \quad \text { for } t \in J \text { and } i=0,1, \ldots n-2
$$

and

$$
\left|u^{(n-1)}(t)\right| \leq C \quad \text { for } t \in J
$$

where $C$ is the constant introduced in Definition 2.1.

To prove Theorem 3.1, firstly, we want to show the following theorems.

Theorem 3.2 There exists at least one solution for BVP (2.18) and (2.19).

Proof By Lemma 2.2, for any $u \in E$, define an operator $\mathcal{H}: E \rightarrow E$ by

$$
\begin{aligned}
\mathcal{H} u(t)= & \frac{1}{(n-2) !} \int_{0}^{t}(t-s)^{n-2} P_{n} u(s) d s \\
& +\sum_{i=0}^{n-2} \frac{t^{i}}{i !}\left(\bar{u}^{[i]}(0)+g_{i}\left(\bar{u}^{[0]}, W_{0} \bar{u}^{[0]}, S_{0} \bar{u}^{[0]}, \ldots, \bar{u}^{[n-2]}, W_{n-2} \bar{u}^{[n-2]},\right.\right. \\
& \left.\left.S_{n-2} \bar{u}^{[n-2]}, \hat{u}^{[n-1]}, W_{n-1} \hat{u}^{[n-1]}, S_{n-1} \hat{u}^{[n-1]}, \bar{u}^{[i]}(0)\right)\right), \quad n \geq 2 .
\end{aligned}
$$

Then we can see that $u(t)$ is a solution of BVP (2.18) and (2.19) if and only if $u(t)$ is a fixed point of $\mathcal{H}$.

Let $\left\{u_{k}\right\}_{k=1}^{\infty} \subseteq E$ with $\left\|u_{k}-u_{0}\right\| \rightarrow 0$ as $k \rightarrow \infty$ in $E$. We want to show that $\| \mathcal{H} u_{k}-$ $\mathcal{H} u_{0} \| \rightarrow 0$ as $k \rightarrow \infty$ in $E$. For $f$ is a Carathéodory function, then it is easy to see $\lim _{k \rightarrow \infty} F\left(t, u_{k}(\cdot)\right)=F\left(t, u_{0}(\cdot)\right)$. By the Lebesgue dominated convergence theorem, $\lim _{k \rightarrow \infty} \int_{0}^{s} F\left(\tau, u_{k}(\cdot)\right) d \tau=\int_{0}^{s} F\left(\tau, u_{0}(\cdot)\right) d \tau$. Let $x_{k}$ be the unique solution of $\mathcal{L}\left(x ; u_{k}\right)=0$, where $\mathcal{L}$ is given by (2.20). In view of (2.17), there exists $r \in L^{1}(0, T)$ such that

$$
\left|F\left(t, u_{k}(\cdot)\right)\right| \leq r(t) \quad \text { on } J^{\prime}
$$

From (2.11), (2.14), (2.21) and the continuity of $g_{n-1}$ and $g_{n-2}$, we see that $g_{u_{k}}$ is bounded and $\lim _{k \rightarrow \infty} g_{u_{k}}=g_{u_{0}}$. Thus, $\left\{x_{k}\right\}$ is bounded. If $\left\{x_{k}\right\}$ is not convergent, then there exist two convergent subsequences $\left\{x_{i_{k}}\right\}$ and $\left\{x_{j_{k}}\right\}$ such that $\lim _{k \rightarrow \infty} x_{i_{k}}=a_{1}, \lim _{k \rightarrow \infty} x_{j_{k}}=a_{2}$. Then, by the continuity of $\varphi^{-1}$ and the Lebesgue dominated convergence theorem again, we have

$$
0=\lim _{k \rightarrow \infty} \mathcal{L}\left(x_{i_{k}} ; u_{i_{k}}\right)=\mathcal{L}\left(a_{1} ; u_{0}\right)
$$

and

$$
0=\lim _{k \rightarrow \infty} \mathcal{L}\left(x_{j_{k}} ; u_{j_{k}}\right)=\mathcal{L}\left(a_{2} ; u_{0}\right),
$$

which contradicts the fact that $\mathcal{L}\left(a_{2} ; u_{0}\right)=\mathcal{L}\left(a_{1} ; u_{0}\right)$. Hence, $\left\{x_{k}\right\}$ is convergent, say $\lim _{k \rightarrow \infty} x_{k}=x_{0}$. Thus, $\mathcal{L}\left(x_{0} ; u_{0}\right)=0$ and $\lim _{k \rightarrow \infty}\left(P_{n} u_{k}\right)(t)=\left(P_{n} u_{0}\right)(t)$. As a consequence, 
we also have

$$
\lim _{k \rightarrow \infty}\left(\mathcal{H} u_{k}\right)^{(j)}(t)=\left(\mathcal{H} u_{0}\right)^{(j)}(t), \quad j=0,1, \ldots, n-1
$$

Thus $\left\|\mathcal{H} u_{k}-\mathcal{H} u_{0}\right\| \rightarrow 0$ as $k \rightarrow \infty$. This shows that $\mathcal{H}: E \rightarrow E$ is continuous.

From (3.3) and the fact that $g_{u}$ is bounded for $u \in E$, this means that $\mathcal{H}$ is uniformly bounded on $E$, and $(\mathcal{H u})^{(j)}(t)$ is equicontinuous on $J$ for $j=0,1, \ldots, n-2$. Now, we show that $(\mathcal{H} u)^{(n-1)}(t)$ is equicontinuous on $J$. From the definition of $\mathcal{H}$ and $P_{n} u(t)$, we have

$$
(\mathcal{H} u)^{(n-1)}(t)=\varphi^{-1}\left(x_{u}-\int_{0}^{t} F(s, u(\cdot)) d s\right) .
$$

Thus, the equicontinuity of $(\mathcal{H} u)^{(n-1)}(t)$ follows from the property of absolute of integrals. By the Arzela-Ascoli theorem, we see that $\mathcal{H}(E)$ is compact. From the Schauder fixed point theorem, $\mathcal{H}$ has at least one fixed point $u \in E$, which is a solution of BVP (2.18) and (2.19). We complete the proof.

Theorem 3.3 If $u(t)$ is a solution of BVP (2.18) and (2.19), then $u(t)$ satisfies (3.1).

Proof We first show that $v^{(n-2)}(t) \leq u^{(n-2)}(t)$ on $J$. To the contrary, suppose that there exists $t^{*} \in J$ such that $v^{(n-2)}\left(t^{*}\right)>u^{(n-2)}\left(t^{*}\right)$. If $t^{*}=0$, then $u^{(n-2)}(0)<v^{(n-2)}(0)$, from (2.19), (H5), (2.12), (2.14), and (2.8), we see that

$$
\begin{aligned}
u^{(n-2)}(0)= & g_{n-2}\left(\bar{u}^{[0]}, W_{0} \bar{u}^{[0]}, S_{0} \bar{u}^{[0]}, \ldots, \bar{u}^{[n-2]}, W_{n-2} \bar{u}^{[n-2]}, S_{n-2} \bar{u}^{[n-2]},\right. \\
& \left.\hat{u}^{[n-1]}, W_{n-1} \hat{u}^{[n-1]}, S_{n-1} \hat{u}^{[n-1]}, \bar{u}^{[n-2]}(0)\right)+\bar{u}^{[n-2]}(0) \\
= & g_{n-2}\left(\bar{u}^{[0]}, W_{0} \bar{u}^{[0]}, S_{0} \bar{u}^{[0]}, \ldots, \bar{u}^{[n-2]}, W_{n-2} \bar{u}^{[n-2]}, S_{n-2} \bar{u}^{[n-2]},\right. \\
& \left.\hat{u}^{[n-1]}, W_{n-1} \hat{u}^{[n-1]}, S_{n-1} \hat{u}^{[n-1]}, v^{(n-2)}(0)\right)+v^{(n-2)}(0) \\
\geq & g_{n-2}\left(v, W_{0} v, S_{0} v, \ldots, v^{(n-2)}, W_{n-2} v^{(n-2)}, S_{n-2} v^{(n-2)},\right. \\
& \left.\hat{u}^{[n-1]}, W_{n-1} \hat{u}^{[n-1]}, S_{n-1} \hat{u}^{[n-1]}, v^{(n-2)}(0)\right)+v^{(n-2)}(0) \\
\geq & \min _{\|z\|_{\infty} \leq C} g_{n-2}\left(v, W_{0} v, S_{0} v, \ldots, v^{(n-2)}, W_{n-2} v^{(n-2)}, S_{n-2} v^{(n-2)},\right. \\
& \left.z, W_{n-1} z, S_{n-1} z, v^{(n-2)}(0)\right)+v^{(n-2)}(0) \\
\geq & v^{(n-2)}(0)
\end{aligned}
$$

which is a contradiction. Similarly, if $t^{*}=T$, then $u^{(n-2)}(T)<v^{(n-2)}(T)$, we have

$$
\begin{aligned}
u^{(n-2)}(T)= & g_{n-1}\left(\bar{u}^{[0]}, W_{0} \bar{u}^{[0]}, S_{0} \bar{u}^{[0]}, \ldots, \bar{u}^{[n-2]}, W_{n-2} \bar{u}^{[n-2]}, S_{n-2} \bar{u}^{[n-2]},\right. \\
& \left.\hat{u}^{[n-1]}, W_{n-1} \hat{u}^{[n-1]}, S_{n-1} \hat{u}^{[n-1]}, \bar{u}^{[n-2]}(T)\right)+\bar{u}^{[n-2]}(T) \\
= & g_{n-1}\left(\bar{u}^{[0]}, W_{0} \bar{u}^{[0]}, S_{0} \bar{u}^{[0]}, \ldots, \bar{u}^{[n-2]}, W_{n-2} \bar{u}^{[n-2]}, S_{n-2} \bar{u}^{[n-2]},\right. \\
& \left.\hat{u}^{[n-1]}, W_{n-1} \hat{u}^{[n-1]}, S_{n-1} \hat{u}^{[n-1]}, v^{(n-2)}(T)\right)+v^{(n-2)}(T) \\
\geq & g_{n-1}\left(v, W_{0} v, S_{0} v, \ldots, v^{(n-2)}, W_{n-2} v^{(n-2)}, S_{n-2} v^{(n-2)}\right. \\
& \left.\hat{u}^{[n-1]}, W_{n-1} \hat{u}^{[n-1]}, S_{n-1} \hat{u}^{[n-1]}, v^{(n-2)}(T)\right)+v^{(n-2)}(T)
\end{aligned}
$$




$$
\begin{aligned}
\geq & \min _{\|z\|_{\infty} \leq C} g_{n-1}\left(v, W_{0} v, S_{0} v, \ldots, v^{(n-2)}, W_{n-2} v^{(n-2)}, S_{n-2} v^{(n-2)},\right. \\
& \left.z, W_{n-1} z, S_{n-1} z, v^{(n-2)}(T)\right)+v^{(n-2)}(T) \\
\geq & v^{(n-2)}(T) .
\end{aligned}
$$

We obtain a contradiction again. Thus, $v^{(n-2)}(0) \leq u^{(n-2)}(0)$ and $v^{(n-2)}(T) \leq u^{(n-2)}(T)$.

Now, if $t^{*} \in J^{\prime}$ such that $v^{(n-2)}\left(t^{*}\right)>u^{(n-2)}\left(t^{*}\right)$. Then $v^{(n-2)}\left(t^{*}\right)-u^{(n-2)}\left(t^{*}\right)>0$. Without loss of generality, we may assume that $v^{(n-2)}\left(t^{*}\right)-u^{(n-2)}\left(t^{*}\right)=\max _{t \in J}\left\{v^{(n-2)}(t)-u^{(n-2)}(t)\right\}$. Then $v^{(n-1)}\left(t^{*}\right)-u^{(n-1)}\left(t^{*}\right)=0$ and there exists a small right neighborhood $\Omega$ of $t^{*}$ such that $v^{(n-2)}(t)-u^{(n-2)}(t)>0$ and $v^{(n-1)}(t) \leq u^{(n-1)}(t)$ for all $t \in \Omega$. We claim that there exists $\tilde{t} \in \Omega$ such that

$$
\left[\varphi\left(v^{(n-1)}(\tilde{t})\right)\right]^{\prime}-\left[\varphi\left(u^{(n-1)}(\tilde{t})\right)\right]^{\prime} \leq 0 .
$$

If this is not true, then $\varphi\left(v^{(n-1)}(t)\right)-\varphi\left(u^{(n-1)}(t)\right)$ is strictly increasing in $\Omega$. Hence, $v^{(n-1)}(t)-u^{(n-1)}(t)>0$ on $\Omega$. This contradicts the assumption that $v^{(n-2)}(t)-u^{(n-2)}(t)$ is maximized at $t^{*}$. Thus, (3.4) holds.

From (2.7), (2.13), and (2.14), we have $\bar{u}^{(n-2)}(\tilde{t})=v^{(n-2)}(\tilde{t})$, also, by (H4), (2.15), and (2.18), we have

$$
\begin{aligned}
& {\left[\varphi\left(v^{(n-1)}(\tilde{t})\right)\right]^{\prime}-\left[\varphi\left(u^{(n-1)}(\tilde{t})\right)\right]^{\prime} } \\
& \geq-f\left(\tilde{t}, v(\tilde{t}), v\left(\alpha_{0}(\tilde{t})\right), W_{0} v(\tilde{t}), S_{0} v(\tilde{t}), \ldots, v^{(n-2)}(\tilde{t}), v^{(n-2)}\left(\alpha_{n-2}(\tilde{t})\right),\right. \\
&\left.W_{n-2} v^{(n-1)}(\tilde{t}), S_{n-2} v^{(n-2)}(\tilde{t}),-C,-C,-W_{n-1} C,-S_{n-1} C\right) \\
&+B u(\tilde{t})+\frac{v^{(n-2)}(\tilde{t})-u^{(n-2)}(\tilde{t})}{1+\left(u^{(n-2)}(\tilde{t})\right)^{2}} \\
& \geq \frac{v^{(n-2)}(\tilde{t})-u^{(n-2)}(\tilde{t})}{1+\left(u^{(n-2)}(\tilde{t})\right)^{2}}>0,
\end{aligned}
$$

which is a contradiction with (3.4). Thus, $v^{(n-2)}(t) \leq u^{(n-2)}(t)$ on $J$. By the same method as above, we can show that $u^{(n-2)}(t) \leq w^{(n-2)}(t)$ on $J$. Hence,

$$
v^{(n-2)}(t) \leq u^{(n-2)}(t) \leq w^{(n-2)}(t) \quad \text { for } t \in J .
$$

Next, we can see that the following inequality holds:

$$
v^{(i)}(0) \leq u^{(i)}(0) \leq w^{(i)}(0), \quad i=0,1, \ldots, n-3 .
$$

In fact, assume there exists $i^{\prime} \in\{0,1, \ldots, n-3\}$ such that $u^{\left(i^{\prime}\right)}(0)<v^{\left(i^{\prime}\right)}(0)$, then, in view of (2.12), $\bar{u}^{\left[i^{\prime}\right]}(0)=v^{\left(i^{\prime}\right)}(0)$. Hence, from (2.19), (H5), (2.8) and (2.12),

$$
\begin{aligned}
u^{\left(i^{\prime}\right)}(0)= & g_{i^{\prime}}\left(\bar{u}^{[0]}, W_{0} \bar{u}^{[0]}, S_{0} \bar{u}^{[0]}, \ldots, \bar{u}^{[n-2]}, W_{n-2} \bar{u}^{[n-2]}, S_{n-2} \bar{u}^{[n-2]},\right. \\
& \left.\hat{u}^{[n-1]}, W_{n-1} \hat{u}^{[n-1]}, S_{n-1} \hat{u}^{[n-1]}, \bar{u}^{\left[i^{\prime}\right]}(0)\right)+\bar{u}^{\left[i^{\prime}\right]}(0) \\
= & g_{i^{\prime}}\left(\bar{u}^{[0]}, W_{0} \bar{u}^{[0]}, S_{0} \bar{u}^{[0]}, \ldots, \bar{u}^{[n-2]}, W_{n-2} \bar{u}^{[n-2]}, S_{n-2} \bar{u}^{[n-2]},\right. \\
& \left.\hat{u}^{[n-1]}, W_{n-1} \hat{u}^{[n-1]}, S_{n-1} \hat{u}^{[n-1]}, v^{\left(i^{\prime}\right)}(0)\right)+v^{\left(i^{\prime}\right)}(0)
\end{aligned}
$$




$$
\begin{aligned}
\geq & g_{i^{\prime}}\left(v^{(0)}, W_{0} v^{(0)}, S_{0} v^{(0)}, \ldots, v^{(n-2)}, W_{n-2} v^{(n-2)}, S_{n-2} v^{(n-2)},\right. \\
& \left.\hat{u}^{[n-1]}, W_{n-1} \hat{u}^{[n-1]}, S_{n-1} \hat{u}^{[n-1]}, v^{\left(i^{\prime}\right)}(0)\right)+v^{\left(i^{\prime}\right)}(0) \\
\geq & \min _{\|z\|_{\infty} \leq C} g_{i^{\prime}}\left(v, W_{0} v, S_{0} v, \ldots, v^{(n-2)}, W_{n-2} v^{(n-2)}, S_{n-2} v^{(n-2)},\right. \\
& \left.z, W_{n-1} z, S_{n-1} z, v^{\left(i^{\prime}\right)}(0)\right)+v^{\left(i^{\prime}\right)}(0) \\
\geq & v^{\left(i^{\prime}\right)}(0) .
\end{aligned}
$$

This is a contradiction. Thus, $v^{(i)}(0) \leq u^{(i)}(0)$ for $i=0,1, \ldots, n-3$. By a similar argument, we see that $u^{(i)}(0) \leq w^{(i)}(0)$ for $i=0,1, \ldots, n-3$. Then (3.6) holds.

Finally, from (3.5) and integral inequality, we have

$$
u^{(n-3)}(t)-v^{(n-3)}(0) \leq u^{(n-3)}(t)-u^{(n-3)}(0) \leq w^{(n-3)}(t)-w^{(n-3)}(0)
$$

and using (3.6), we obtain $v^{(n-3)}(t) \leq u^{(n-3)}(t) \leq w^{(n-3)}(t)$. Similarly, we can show that $u(t)$ satisfies (3.1). The proof is completed.

Theorem 3.4 If $u(t)$ is a solution of $B V P(2.18)$ and (2.19), then $u^{(n-1)}(t)$ satisfies (3.2).

Proof From Theorem 3.3, we know that $u(t)$ satisfies (3.1). If (3.2) does not hold, then there exists $t_{0} \in J$ such that $u^{(n-1)}\left(t_{0}\right)>C$ or $u^{(n-1)}\left(t_{0}\right)<-C$. By the mean value theorem, there exists $\tilde{t} \in J$ such that $T u^{(n-1)}(\tilde{t})=u^{(n-2)}(T)-u^{(n-2)}(0)$. Then, from (2.2), (2.3), and (2.17), we see that

$$
-C<-\xi \leq \frac{v^{(n-2)}(T)-w^{(n-2)}(0)}{T} \leq u^{(n-1)}(\tilde{t}) \leq \frac{w^{(n-2)}(T)-v^{(n-2)}(0)}{T} \leq \xi<C .
$$

If $u^{(n-1)}\left(t_{0}\right)>C$ there exist $t_{1}, t_{2} \in J$ such that $u^{(n-1)}\left(t_{1}\right)=\xi, u^{(n-1)}\left(t_{2}\right)=C$ and

$$
\xi=u^{(n-1)}\left(t_{1}\right) \leq u^{(n-1)}(t) \leq u^{[n-1]}\left(t_{2}\right)=C \text { for } t \in I,
$$

where $I=\left[t_{1}, t_{2}\right]$ or $I=\left[t_{2}, t_{1}\right]$. In the following, we only consider the case $I=\left[t_{1}, t_{2}\right]$, since the other case can be treated similarly. From (2.14) and (3.7), $\hat{u}^{(n-1)}(t)=u^{(n-1)}(t)$ on $I$, and in view of (2.11) and (3.1), we have $\bar{u}^{[i]}(t)=u^{(i)}(t)$ for $t \in J$ and $i=0,1, \ldots, n-2$. Thus, from (2.15),

$$
\begin{aligned}
F(t, u(\cdot))= & f\left(t, u(t), u\left(\alpha_{0}(t)\right), W_{0} u(t), S_{0} u(t), \ldots, W_{n-2} u^{(n-2)}(t), S_{n-2} u^{(n-2)}(t),\right. \\
& \left.u^{(n-1)}(t), \hat{u}^{(n-1)}\left(\alpha_{n-1}(t)\right), W_{n-1} \hat{u}^{(n-1)}(t), S_{n-1} \hat{u}^{(n-1)}(t)\right), \quad t \in I .
\end{aligned}
$$

Then, by a change of variables and from (2.4) and (2.18), we can obtain

$$
\begin{aligned}
& \int_{\phi(\xi)}^{\phi(C)} \frac{\left(\phi^{-1}(x)\right)^{(p-1) / p}}{\psi\left(\left|\phi^{-1}(x)\right|\right)} d x \\
& \quad=\int_{\phi\left(u^{(n-1)}\left(t_{1}\right)\right)}^{\phi\left(u^{(n-1)}\left(t_{2}\right)\right)} \frac{\left.\left(\phi^{-1}(x)\right)\right)^{(p-1) / p}}{\psi\left(\left|\phi^{-1}(x)\right|\right)} d x \\
& \quad=\int_{t_{1}}^{t_{2}} \frac{\left[\phi\left(u^{(n-1)}(s)\right)\right]^{\prime}}{\psi\left(\left|u^{(n-1)}(s)\right|\right)}\left(u^{(n-1)}(s)\right)^{(p-1) / p} d s
\end{aligned}
$$




$$
\begin{aligned}
& =\int_{t_{1}}^{t_{2}} \frac{\left(\varphi\left(u^{(n-1)}(s)\right)\right)^{\prime}}{\psi\left(\left|u^{(n-1)}(s)\right|\right)}\left(u^{(n-1)}(s)\right)^{(p-1) / p} d s \\
& =\int_{t_{1}}^{t_{2}} \frac{-f\left(s, u(s), \ldots, S_{n-1} u^{(n-1)}(s)\right)}{\psi\left(\left|u^{(n-1)}(s)\right|\right)}\left(u^{(n-1)}(s)\right)^{(p-1) / p} d s \\
& \leq \int_{t_{1}}^{t_{2}} \vartheta(s)\left(u^{(n-1)}(s)\right)^{(p-1) / p} d s .
\end{aligned}
$$

Hence, the Hölder inequality implies

$$
\begin{aligned}
\int_{\phi(\xi)}^{\phi(C)} \frac{\left(\phi^{-1}(x)\right)^{(p-1) / p}}{\psi\left(\left|\phi^{-1}(x)\right|\right)} d x & \leq\|\vartheta\|_{p}\left(\int_{t_{1}}^{t_{2}} u^{(n-1)}(s) d s\right)^{(p-1) / p} \\
& =\|\vartheta\|_{p}\left(u^{(n-2)}\left(t_{2}\right)-u^{(n-2)}\left(t_{1}\right)\right)^{(p-1) / p} \\
& \leq\|\vartheta\|_{p}\left(\max _{t \in J} w^{(n-2)}(t)-\min _{t \in J} v^{(n-2)}(t)\right)^{(p-1) / p} \\
& =\|\vartheta\|_{p} \zeta^{(p-1) / p},
\end{aligned}
$$

where $\zeta$ is defined by (2.6). But this contradicts with (2.5). Therefore, $u^{(n-1)}(t) \leq C$. If $u^{(n-1)}(t)<-C$, by a similar argument as above, we can show that (3.2) holds. Hence the proof of the theorem is completed.

Now we are in a position to prove Theorem 3.1.

Proof of Theorem 3.1 Note that any solution of BVP (2.18) and (2.19) satisfying (3.1), (3.2) is a solution of BVP (1.1) and (1.2). The conclusion readily follows from Theorem 3.2-3.4.

\section{Example}

Example 4.1 Consider the boundary value problem consisting of the equation

$$
\begin{aligned}
& \left(\left(u^{\prime \prime}(t)\right)^{3}\right)^{\prime}+\frac{1}{15} t^{-\frac{1}{2}}(1+u(t))-\frac{t}{500}\left[t^{3}-\int_{0}^{t} t s u(s) d s\right]^{3} \\
& -\frac{1}{300} t^{2}\left[t-u\left(t^{3}\right)\right]^{3}+4 t^{-\frac{1}{2}}\left[\int_{0}^{1}(t+s) u^{\prime \prime}(s) d s\right]^{3}=0, \quad t \in(0,1),
\end{aligned}
$$

and the boundary condition

$$
\left\{\begin{array}{l}
\min _{s \in[0,1]} u^{\prime}(s)-6 u(0)+\frac{1}{2}=0 \\
\int_{0}^{t}(t s) u(s) d s-4 e^{2} u^{\prime}(0)=0 \\
u^{\prime \prime}\left(\frac{1}{4}\right)-2 e^{2} u^{\prime}(1)=0
\end{array}\right.
$$

BVP (4.1) and (4.2) has at least one solution $u(t)$ satisfying

$$
\begin{aligned}
& -(t+1)^{2} \leq u(t) \leq(t+1)^{2}, \\
& -2(t+1) \leq u^{\prime}(t) \leq 2(t+1),
\end{aligned}
$$


and

$$
-12 e^{2} \leq u^{\prime \prime}(t) \leq 12 e^{2}
$$

for $t \in[0,1]$.

In fact, if we let $n=3, \phi(x)=x^{3}$,

$$
f\left(t, x_{0}, x_{1}, \ldots, x_{11}\right)=\frac{1}{15} t^{-\frac{1}{2}}\left(1+x_{0}\right)-\frac{t}{500}\left[t^{3}-x_{2}\right]^{3}-\frac{1}{300} t^{2}\left[t-x_{5}\right]^{3}+4 t^{-\frac{1}{2}} x_{11}^{3},
$$

for $\left(x_{0}, x_{1}, \ldots, x_{11}\right) \in R^{4 n}$, and $\beta_{0}(t)=t, k_{0}(t, s)=t s, \alpha_{1}(t)=t^{3}, h_{2}(t, s)=t+s, H=\max \left\{h_{2}(t, s)\right.$ : $(t, s) \in[0,1] \times[0,1]\}=2$, and

$$
\begin{aligned}
& g_{0}\left(y_{0}, \ldots, y_{8}, z\right)=\min _{s \in[0,1]} y_{3}(s)-6 z+\frac{1}{2}, \\
& g_{1}\left(y_{0}, \ldots, y_{8}, z\right)=y_{1}(t)-4 e^{2} z, \\
& g_{2}\left(y_{0}, \ldots, y_{8}, z\right)=y_{6}\left(\frac{1}{4}\right)-2 e^{2} z
\end{aligned}
$$

for $\left(y_{0}, \ldots, y_{8}, z\right) \in(C[0,1])^{9} \times R$, then it is easy to see that BVP (4.1) and (4.2) is of the form of BVP (1.1) and (1.2). Clearly, (H1), (H2) and (H5) hold.

Let $v(t)=-(t+1)^{2}$ and $w(t)=(t+1)^{2}$. Obviously, $v(t), w(t)$ satisfy (2.1). Define $\vartheta(t)=$ $\frac{1}{4} t^{-1 / 2}$ and $\psi(x)=9+16 x^{3}$. Then $\vartheta \in L^{1}(0,1)$ with $\|\vartheta\|_{1}=\frac{1}{2}, \psi(x)>0$ on $[0, \infty)$, and

$$
\left|f\left(t, x_{0}, x_{1}, \ldots, x_{11}\right)\right| \leq \frac{1}{4} t^{-\frac{1}{2}}\left(9+16\left|x_{11}\right|^{3}\right)=\vartheta(t) \psi\left(\left|x_{11}\right|\right)
$$

on $(0,1) \times \mathbb{D}_{v}^{w} \times R^{4}$, where $\mathbb{D}_{v}^{w}$ is given by Definition 2.1 . Thus (2.4) holds. For $\xi$ defined by (2.2), we have $\xi=6$ with $C=12 e^{2}$ and $p=1$, and it is easy to check that (2.3) holds. Through computations, we can obtain (2.5). Hence, $f$ satisfies the Nagumo condition with respect to $v, w$ i.e. (H3) holds. Moreover, a simple computation shows that $v(t)$ and $w(t)$ satisfy (2.7)-(2.10). Hence (H2) holds. Finally, obviously (H4) holds.

Therefore, by Theorem 3.1, BVP (4.1) and (4.2) has at least one solution $u(t)$ satisfying (4.3)-(4.5).

\section{Conclusions}

In this paper, we obtain a new existence result for higher-order integro-differential BVPs with $\phi$-Laplacian like operator and functional boundary conditions. Firstly, we state some preliminaries and lemmas such as the definitions if we have the Nagumo condition and a pair of coupled lower and upper solutions. Secondly, under conditions (H1)-(H5), we get the main result (Theorem 3.1), and we prove the result in three steps (Theorems 3.23.4) which mainly use lower and upper solutions and the Schauder fixed point theorem. Finally, an example is given to illustrate our main result.

The authors declare that they have no competing interests. 


\section{Acknowledgements}

This work was supported by Natural Science Foundation of China Grant No. 11461021, Natural Science Foundation of Guangxi Grant No. 2014GXNSFDA1 18002, Scientific Research Foundation of Guangxi Education Department No. ZD2014131, No. 2013YB236, the open fund of Guangxi Key Laboratory of Hybrid Computation and IC Design Analysis No. HCIC201305 and the Scientific Research Project of Hezhou University No. 2014ZC13. The authors wish to thank the anonymous reviewers for their helpful comments and suggestions.

\section{Received: 24 June 2014 Accepted: 22 October 2014 Published: 04 Nov 2014}

\section{References}

1. Lakshmikantham, V: Some problems in integro-differential equations of Volterra type. J. Integral Equ. 10, 137-146 (1985)

2. Guo, D, Lakshmikantham, V, Liu, XZ (ed.): Nonlinear Integral Equations in Abstract Spaces. Kluwer Academic, Dordrecht (1996)

3. Shi, GL, Meng, XR: Monotone iterative for fourth-order p-Laplacian boundary value problems with impulsive effects. Appl. Math. Comput. 181, 1243-1248 (2006)

4. Cabada, A, Otero-Espinar, V: Existence and comparison results for difference $\phi$-Laplacian boundary value problems with lower and upper solutions in reverse order. J. Math. Anal. Appl. 267, 501-521 (2002)

5. Misawa, M: A Hölder estimate for nonlinear parabolic systems of $p$-Laplacian type. J. Differ. Equ. 254, 847-878 (2013)

6. Agarwal, RP: On fourth order boundary value problems arising in beam analysis. Differ. Integral Equ. 2, 91-110 (1989)

7. Wei, Z: Existence of positive solutions for $n$ th-order $p$-Laplacian singular sublinear boundary value problems. Appl. Math. Lett. 36, 25-30 (2014)

8. Davis, JD, Henderson, J, Wong, PJY: General Lidstone problems: multiplicity and symmetry of solutions. J. Math. Anal. Appl. 251, 527-548 (2000)

9. Fialho, JF, Minhós, F: Higher order functional boundary value problems without monotone assumptions. Bound. Value Probl. 2013, Article ID 81 (2013)

10. Agarwal, RP: Boundary Value Problems for Higher Order Differential Equations. World Scientific, Singapore (1986)

11. Lee, EK, Lee, YH: Multiple positive solutions of singular two points boundary value problems for second order impulsive differential equations. Appl. Math. Comput. 158, 745-759 (2004)

12. Eloe, PW, Ahmad, B: Positive solutions of a nonlinear $n$th order boundary value problem with nonlocal conditions Appl. Math. Lett. 18, 521-527 (2005)

13. Mawhin, J: Homotopy and nonlinear boundary value problems involving singular $\phi$-Laplacians. J. Fixed Point Theory Appl. 13, 25-35 (2013)

14. Graef, JR, Yang, B: Positive solutions to a multi-point higher order boundary value problems. J. Math. Anal. Appl. 316 409-421 (2006)

15. Graef, JR, Kong, L, Kong, Q: Higher order multi-point boundary value problems. Math. Nachr. 284, 39-52 (2011)

16. Wang, DB, Guan, W: Multiple positive solutions for third-order $p$-Laplacian functional dynamic equations on time scales. Adv. Differ. Equ. 2014, Article ID 145 (2014)

17. Graef, JR, Kong, L, Minhós, FM: Higher order boundary value problems with $\phi$-Laplacian and functional boundary conditions. Comput. Math. Appl. 61, 236-249 (2011)

18. Graef, JR, Kong, L: Existence of solutions for nonlinear boundary value problems. Commun. Appl. Nonlinear Anal. 14 39-60 (2007)

19. Bai, DY: A global result for discrete $\phi$-Laplacian eigenvalue problems. Adv. Differ. Equ. 2013, Article ID 264 (2013)

20. Wang, W, Yang, X, Shen, J: Boundary value problems involving upper and lower solutions in reverse order. J. Comput. Appl. Math. 230, 1-7 (2009)

21. Ehme, J, Eloe, PW, Henderson, J: Upper and lower solution methods for fully nonlinear boundary value problems. J. Differ. Equ. 180, 51-64 (2002)

22. Franco, D, Regan, DO, Perán, J: Fourth-order problems with nonlinear boundary conditions. J. Comput. Appl. Math. $174,315-327$ (2005)

23. Cabada, A, Pouso, R, Minhós, F: Extremal solutions to fourth-order functional boundary value problems including multipoint conditions. Nonlinear Anal. 10, 2157-2170 (2009)

24. Henderson, J: Solutions of multipoint boundary value problems for second order equations. Dyn. Syst. Appl. 15 , 111-117 (2006)

25. Cabada, A, Pouso, RL: Existence results for the problem $\left(\phi\left(u^{\prime}\right)\right)^{\prime}=f\left(t, u, u^{\prime}\right)$ with nonlinear boundary conditions. Nonlinear Anal. 35, 221-231 (1999)

26. Kong, L, Kong, Q: Second-order boundary value problems with nonhomogeneous boundary conditions (I). Math. Nachr. 278, 173-193 (2005)

10.1186/1687-1847-2014-285

Cite this article as: Guo et al.: BVPs for higher-order integro-differential equations with $\phi$-Laplacian and functional boundary conditions. Advances in Difference Equations 2014, 2014:285 